\title{
Brasil Sorridente: reconhecendo a história para reforçar a constante luta pela equidade em Odontologia
}

\author{
Smiling Brazil: recognizing history to reinforce the constant struggle for equity in Dentistry \\ Brasil Sonriente: reconocer la historia para reforzar la lucha constante por la equidad en \\ Odontología
}

Recebido: 26/07/2021 | Revisado: 30/07/2021 | Aceito: 30/07/2021 | Publicado: 05/08/2021

\author{
Gerson Aparecido Foratori-Junior \\ ORCID: https://orcid.org/0000-0003-4760-8948 \\ Universidade de São Paulo, Brasil \\ E-mail: gerson.foratori@usp.br \\ Gilberto Alfredo Pucca Junior \\ ORCID: https://orcid.org/0000-0002-8781-9857 \\ Universidade de Brasília, Brasil \\ E-mail: gilberto.pucca@hotmail.com
}

\begin{abstract}
Resumo
A Constituição de 1988 representou um marco na gestão da saúde no Brasil, fato este que também refletiu nas práticas de saúde bucal. Os profissionais do setor odontológico sempre ficaram às margens do sistema de saúde no Brasil. No início da década de 2000, com base na nova Política Nacional de Saúde Bucal, o "Brasil Sorridente” foi criado, o qual teve como as principais linhas de ação: a reorganização da atenção primária em saúde bucal, com a implementação e ampliação das equipes de saúde bucal na Estratégia Saúde da Família; e ampliação e qualificação da Atenção Especializada. O processo de trabalho em saúde bucal passou por uma reorganização, tendo foco na interdisciplinaridade, no multiprofissionalismo e na intersetorialidade. Dentre as ações de promoção em saúde e prevenção às doenças destacam-se àquelas relacionadas à fluoretação das águas, educação em saúde (alimentação saudável, redução do tabagismo, higiene bucal adequada etc), higiene bucal supervisionada, aplicação tópica de flúor e medidas ativas de prevenção à cárie dentária, doença periodontal e câncer bucal. O inquérito nacional "SB Brasil 2010" mostrou resultados positivos da implementação do "Brasil Sorridente". Nesse exame epidemiológico, o Brasil passou integrar o grupo de países com baixo índice de cárie dentária, com base nos dados do índice de cárie aos 12 anos. As principais dificuldades enfrentadas por essa política referem-se à gestão precária e ao baixo financiamento. A pandemia do novo coronavírus e as estratégias adotadas pelo governo brasileiro para a minimizar as consequências dessa doença também refletiram para o aumento das iniquidades em saúde bucal. É importante aprofundarmos os nossos conhecimentos sobre a política "Brasil Sorridente" e nos atualizarmos de acordo ao contexto político-social em que vivemos, para sermos ativos na luta para maior ampliação do acesso e qualificação dessa política. Palavras-chave: Atenção à saúde; Inquéritos de saúde bucal; Serviços de vigilância epidemiológica.
\end{abstract}

\begin{abstract}
The 1988 Constitution represented a milestone in health management in Brazil, a fact that also reflected in oral health practices. Professionals in the dental sector have always been on the margins of the health system in Brazil. In the early 2000s, based on the new National Oral Health Policy, "Brasil Sorridente"/“Smiling Brazil" was created, whose main lines of action were: the reorganization of primary care in oral health, with the implementation and expansion of oral health teams in the Family Health Strategy; and expansion and qualification of Specialized Care. The work process in oral health underwent a reorganization, focusing on interdisciplinarity, multidisciplinarity and intersectoriality. Among the health promotion and disease prevention actions, those related to water fluoridation, health education (healthy nutritional behaviors, smoking reduction, proper oral hygiene etc), supervised oral hygiene, topical application of fluoride and active measures of prevention of dental caries, periodontal disease and oral cancer. The national survey "SB Brasil 2010" showed positive results from the implementation of "Brasil Sorridente"/“Smiling Brazil". In this epidemiological examination, Brazil became part of the group of countries with a low rate of dental caries, based on data from the caries index at 12 years of age. The main difficulties faced by this policy refer to poor management and low funding. The new coronavirus pandemic and the strategies adopted by the Brazilian government to minimize the consequences of this disease also reflected in the increase of inequities in oral health. It is important to deepen our knowledge of the "Smiling Brazil" policy and update ourselves according to the political-social context in which we live, in order to be active in the fight for greater expansion of access and qualification of this policy.
\end{abstract}

Keywords: Delivery of health care; Dental health surveys; Epidemiologic surveillance services. 


\begin{abstract}
Resumen
La Constitución de 1988 representó un hito en la gestión de la salud en Brasil, hecho que también se reflejó en las prácticas de salud bucal. Los profesionales del sector dental siempre han estado al margen del sistema de salud en Brasil. A principios de la década del 2000, a partir de la nueva Política Nacional de Salud Bucal, se crea Brasil Sorridente, cuyas principales líneas de acción fueron: la reorganización de la atención primaria en salud bucal, con la implementación y ampliación de equipos de salud bucal en la Estrategia Salud de la Familia; y expansión y calificación de Atención Especializada. El proceso de trabajo en salud bucal sufrió una reorganización, enfocándose en la interdisciplinariedad, multidisciplinariedad e intersectorialidad. Entre las acciones de promoción de la salud y prevención de enfermedades, las relacionadas con la fluoración del agua, la educación sanitaria (alimentación saludable, reducción del tabaquismo, higiene bucal adecuada, etc.), higiene bucal supervisada, aplicación tópica de flúor y medidas activas de prevención de caries, enfermedades periodontales y cáncer. La encuesta nacional "SB Brasil 2010" arrojó resultados positivos de la implementación de Brasil Sonriente. En este examen epidemiológico, Brasil pasó a formar parte del grupo de países con una baja tasa de caries dental, según los datos del índice de caries a los 12 años. Las principales dificultades a las que se enfrenta esta política se refieren a una mala gestión y escasa financiación. La nueva pandemia de coronavirus y las estrategias adoptadas por el gobierno brasileño para minimizar las consecuencias de esta enfermedad también se reflejaron en el aumento de las inequidades en salud bucal. Es importante para nosotros profundizar en el conocimiento de la política "Brasil Sonriente" y actualizarnos de acuerdo al contexto político-social en el que vivimos, para estar activos en la lucha por una mayor expansión del acceso y calificación de esta política.
\end{abstract}

Palabras clave: Atención a la salud; Encuestas de salud bucal; Servicios de vigilancia epidemiológica.

\title{
Prezados editores,
}

A Constituição de 1988 representou um marco na gestão da saúde no Brasil, a qual passou a ser considerada um direito de todos e um dever do Estado (Silva \& Silva, 2020). Fato este que também refletiu nas práticas de saúde bucal. Os anos antecessores da Constituição de 1988 foram marcados pela recuperação das liberdades democráticas, florescimento e multiplicação dos movimentos sociais em busca de melhores condições de vida (Paim, et al., 2011).

No âmbito da Odontologia, o modelo das práticas odontológicas caracterizado pelo traço iatrogênico e mutilador, além do caráter de monopólio, onde as atividades eram centradas exclusivamente nos cirurgiões-dentistas, começou a receber diversas críticas dos profissionais e estudiosos da área. Ademais, a profissão apresentava uma tradição fortemente liberalprivatista, o que gerou discussão na época sobre a necessidade de alinhar um novo modelo de prática odontológica às propostas mais gerais do movimento da Reforma Sanitária que foram formuladas naquele período. Afinal, embora o trabalho realizado nas clínicas odontológicas seja relevante e resolva os problemas de forma pontual, ele não é acessível a todos, não sendo, portanto, suficientemente capaz de produzir a saúde bucal em termos populacionais, visto que a saúde resulta de uma gama de fatores/determinantes bio-psíquico-sociais que vão além das variáveis biológicas (Paim, et al., 2011).

Os profissionais do setor odontológico sempre ficaram às margens do sistema de saúde no Brasil. A partir da década de 50, houve a hegemonia do Sistema Incremental, um sistema no qual a saúde bucal de crianças em idade escolar foi priorizada, diferentemente do que ocorreu com outras faixas etárias, que acabaram sendo excluídas da atenção odontológica pública (Nickel, Lima \& Bidigaray da Silva, 2008).

Na $1^{a}$ Conferência Nacional de Saúde Bucal, em 1986, foram aprovadas deliberações que tinham como proposta a inserção da saúde bucal em um Sistema Único de Saúde por meio de um Programa Nacional de Saúde Bucal com base nas diretrizes da área, universalizado, hierarquizado, regionalizado e descentralizado, com a municipalização dos serviços e fortalecimento do poder decisório municipal. Apesar dos debates no Congresso Nacional, os governos subsequentes não colocaram em prática tais deliberações, o que expressava os conflitos do período.

Posteriormente, a partir do ano de 1990, não houve a explicitação de uma política nacional voltada à saúde bucal. Foram registradas apenas metas voltadas às atividades de prevenção ao câncer de orofaringe, cárie dentária e doença periodontal. Nesse contexto, reforçavam-se os modelos de atenção à saúde bucal que valorizavam a quantidade de procedimentos executados, resultando em superposição de ações, desperdícios de recursos e mau atendimento à população. Os 
governos subsequentes também não deram prioridade à Política Nacional de Saúde Bucal.

Em 2000, o Instituto Brasileiro de Geografia e Estatística (IBGE) publicou os dados da PNAD-1988 (Pesquisa Nacional por Amostra de Domicílios), no qual indicava que quase $20 \%$ da população brasileira nunca havia ido ao dentista. Frente ao longo processo de discussões que já envolvia as entidades odontológicas e aos preocupantes dados da PNAD-1988, o governo da época possibilitou a inserção da equipe de saúde bucal no Programa de Saúde da Família (Lourenço, et al, 2009).

Sendo assim, no início da década de 2000, com base na nova Política Nacional de Saúde Bucal resultante dos debates da 3a Conferência de Saúde Bucal, o "Brasil Sorridente" foi criado, o qual teve como as principais linhas de ação: a reorganização da atenção primária em saúde bucal, com a implementação e ampliação das equipes de saúde bucal na Estratégia Saúde da Família; e ampliação e qualificação da Atenção Especializada (Pucca, et al., 2015). Previamente à criação do Brasil Sorridente, como mencionado, a Odontologia pública Brasileira expressava seu caráter materno-infantil, que não era suficiente para atender às demandas epidemiológicas da população naquele contexto.

Desta forma, o processo de trabalho em saúde bucal passou por uma reorganização, tendo foco na interdisciplinaridade, no multiprofissionalismo e na intersetorialidade. A atuação da equipe de saúde bucal não se limitou exclusivamente ao campo biológico ou ao trabalho técnico-odontológico, mas passou a interagir com profissionais de outras áreas, de forma a ampliar seu conhecimento, permitindo a abordagem do indivíduo em seu contexto mais amplo, atentando-se ao contexto socioeconômico-cultural no qual ele está inserido. Ademais, a equipe se adequa de modo a alcançar a integralidade da atenção em saúde bucal através das redes de atenção (Gabriel, et al., 2020), visto que passa a oferecer de forma conjunta as ações de promoção de saúde, prevenção de doenças, tratamento, cura e reabilitação, tanto no nível individual quanto coletivo.

Dentre as ações de promoção em saúde e prevenção às doenças destacam-se àquelas relacionadas à fluoretação das águas, educação em saúde (alimentação saudável, redução do tabagismo, higiene bucal adequada etc), higiene bucal supervisionada, aplicação tópica de flúor e medidas ativas de prevenção à cárie dentária, doença periodontal e câncer bucal. Em relação às ações de recuperação, dá-se foco ao diagnóstico e tratamento das doenças, priorizando os tratamentos mais conservadores, afim de reduzir a realidade de ações mutiladores que foram realizadas por muito tempo na Odontologia. Por fim, as ações de recuperação baseiam-se na recuperação parcial ou total dos elementos dentários ausentes.

Tendo como objetivo a ampliação e qualificação da atenção primária, essa política garantiu ampliação do acesso da população aos cuidados bucais, sendo a atenção básica responsável pela resolução de um grande número de problemas, ainda que eles fossem de maior complexidade (Pucca Junior, et al., 2020). Portanto, a inclusão de tratamentos de reabilitação protética em Unidades Básicas de Saúde tornou-se uma realidade em diversas cidades ao redor do país.

Mais de R\$ 6 bilhões foram investidos em saúde bucal no Brasil nos primeiros 10 anos da implantação do "Brasil Sorridente". Esse investimento foi realizado em diversas áreas, incluindo o financiamento de estudos científicos e a formação de membros da equipe de saúde bucal (dentistas, técnicos em saúde bucal e auxiliares de saúde bucal) (Antoniassi, et al., 2021; Pucca, et al., 2015). A partir do "Brasil Sorridente", o aumento no número de cirurgiões-dentistas atuando no SUS foi significativo, sendo que um quarto de todos os dentistas brasileiros passaram a ter vínculo com o setor público de saúde (Gabriel, et al., 2020; Pucca, et al., 2015).

O inquérito nacional "SB Brasil 2010" mostrou resultados positivos da implementação do "Brasil Sorridente”. Nesse exame epidemiológico, o Brasil passou integrar o grupo de países com baixo índice de cárie dentária, com base no índice de cárie aos 12 anos. A redução da cárie dentária também ocorreu para crianças aos 5 anos, bem como para os adolescentes (1219 anos) e adultos (35-44 anos) (Brasil, 2010; Checchi, et al., 2021). As divergências regionais e o edentulismo ainda alarmante dentre os idosos persistiam como principais barreiras a serem ultrapassadas por essa política de saúde bucal.

As principais dificuldades enfrentadas por essa política referem-se à gestão precária e ao baixo financiamento (Damasceno, Cruz, \& Barros, 2021). A gestão partidarizada, com excessivos cargos de confiança e baixa prioridade da pauta 
em questão nas políticas de saúde, nos âmbitos federal, governamentais e municipais enfraquecem o modelo de atenção universal, integralizado e equitativo preconizado pela política "Brasil Sorridente". A redução drástica do investimento no setor odontológico nos últimos anos, resultante não apenas da crise econômica do país, mas também da crise política, afeta os princípios norteadores dessa Política Nacional de Saúde Bucal e inviabiliza a ampliação e qualificação dos serviços básicos e especializado (Lima, et al., 2021; Narvai, 2020).

Somadas às dificuldades acima mencionadas, a pandemia do novo coronavírus (SARS-CoV-2) e as estratégias adotadas pelo governo brasileiro para a minimizar as consequências dessa doença também refletiram para que o acesso da população aos serviços de saúde bucal ficasse comprometido, reafirmando o aumento das diferenças sociais e, consequentemente, iniquidades em saúde no país (Castro, et al., 2020; Ribeiro, et al., 2021). Pesquisas futuras de base populacional devem ser conduzidas a fim de evidenciar o impacto da pandemia nas condições de saúde bucal e no acesso aos serviços de saúde da população brasileira.

É importante aprofundarmos os nossos conhecimentos sobre a política "Brasil Sorridente" e nos atualizarmos de acordo ao contexto político-social em que vivemos, pois, a partir do conhecimento histórico da implementação dessa política, bem como dos seus resultados alcançados, podemos continuar ativos na luta para maior ampliação do acesso e qualificação dessa política que visa reduzir as iniquidades em saúde bucal da população brasileira.

\section{Referências}

Antoniassi, C. P., Barcellos, C., Siqueira, J. de O., \& Fujimaki, M. (2021). Diffusion of the educational process for the qualification of SUS management in oral health: A spatial analysis. Research, Society and Development, 10(8), e34410817460. https://doi.org/10.33448/rsd-v10i8.17460.

Brasil (2012). Ministério da Saúde. Secretaria de Atenção à Saúde. Secretaria de Vigilância em Saúde. SB Brasil 2010: Pesquisa Nacional de Saúde Bucal: resultados principais. Brasília: Ministério da Saúde.

Castro, C. S. de, Holzgrefe Júnior, J. V., Reis, R. B., Andrade, B. B., \& Quintanilha, L. F. (2020). COVID-19 pandemic: scenario of the Brazilian health system for coping with the crisis. Research, Society and Development, 9(7), e516974383. https://doi.org/10.33448/rsd-v9i7.4383.

Checchi, M. H. R. de, Tenani, C. F., Morais, F. D. M. G. de, Meneghim, M. de C., \& Pereira, A. C. (2021). Compilation of epidemiological surveys in oral health in Brazil from 1986 to 2015: Main methodological aspects. Research, Society and Development, 10(8), e53410817614. https://doi.org/10.33448/rsdv10i8.17614.

Damasceno, K. S. M., Cruz, D. N., \& Barros, S. G. de. (2021). Accessibility to dental services in SUS: a literature review. Research, Society and Development, 10(3), e17610313194. https://doi.org/10.33448/rsd-v10i3.13194.

Gabriel, M., Cayetano, M. H., Chagas, M. M., Araujo, M. E., Dussault, G., Pucca Junior, G. A., \& Almeida, F. (2020). Admission of dentist in Brazilian Universal Health System (SUS): a priority agenda for the strengthening of Smiling Brazil. Mecanismos de ingresso de dentistas no SUS: uma agenda prioritária para o fortalecimento do Brasil Sorridente. Ciencia \& saude coletiva, 25(3), 859-868. https://doi.org/10.1590/1413-81232020253.14532018.

Lima, E. B., Melo, R. B., Sá, C. D. L., Cruz, K. R. S., Moraes, R. P., \& Bernardino, R. de S. (2021). Evaluation of the supply and production of dental specialties in public secondary care services in a state in northeastern Brazil. Research, Society and Development, 10(6), e59410616236. https://doi.org/10.33448/rsd-v10i6.16236.

Lourenço, E., Silva, A. C., Meneghin, M., \& Pereira, A. C. (2009). A inserção de equipes de saúde bucal no Programa Saúde da Família no Estado de Minas Gerais [The insertion of oral health services in the Family Health Program at Minas Gerais State, Brazil]. Ciencia \& saude coletiva, 14 Suppl 1, $1367-1377$. https://doi.org/10.1590/s1413-81232009000800009.

Narvai, P. C. (2020). Ocaso do 'Brasil Sorridente' e perspectivas da Política Nacional de Saúde Bucal em meados do século XXI. Tempus - Actas De Saúde Coletiva, 14(1), 175-187. https://doi.org/10.18569/tempus.v14i1.2622.

Nickel, D. A., Lima, F. G., \& Bidigaray da Silva, B. (2008). Modelos assistenciais em saúde bucal no Brasil [Dental care models in Brazil]. Cadernos de saude publica, 24(2), 241-246. https://doi.org/10.1590/s0102-311x2008000200002.

Paim, J., Travassos, C., Almeida, C., Bahia, L., \& Macinko, J. (2011). The Brazilian health system: history, advances, and challenges. Lancet, 377(9779), 1778-1797. https://doi.org/10.1016/S0140-6736(11)60054-8

Pucca, G. A., Jr, Gabriel, M., de Araujo, M. E., \& de Almeida, F. C. (2015). Ten Years of a National Oral Health Policy in Brazil: Innovation, Boldness, and Numerous Challenges. Journal of dental research, 94(10), 1333-1337. https://doi.org/10.1177/0022034515599979.

Pucca Junior, G. A., Gabriel, M., Almeida Carrer, F. C. de, Paludetto Junior, M., Lucena, E. H. G. de, \& Melo, N. S. de. (2020). Acesso e cobertura populacional à saúde bucal após a implementação da Política Nacional de Saúde Bucal "Brasil Sorridente". Tempus - Actas De Saúde Coletiva, 14(1), 29-43. https://doi.org/10.18569/tempus.v14i1.2629. 
Research, Society and Development, v. 10, n. 10, e75101018745, 2021

(CC BY 4.0) | ISSN 2525-3409 | DOI: http://dx.doi.org/10.33448/rsd-v10i10.18745

Ribeiro, L. M. C. de A. V., Ferreira, M. M., Lima, J. G. da C., Farias, D. M., Santos, A. A., Medeiros, C. K. S., Almeida, D. R. de M. F., Gonçalves, G. C., Silva, H. F. V., Araújo, S. L. S., Pinheiro, J. C., Leite, R. B., \& Oliveira, R. D. B. de . (2021). The impact of the COVID-19 pandemic on children's dental care in the Unique Health System of João Pessoa - PB. Research, Society and Development, 10(5), e17110515089. https://doi.org/10.33448/rsd-v10i5.15089.

Silva, L. F. de A., \& Silva, D. A. da. (2020). The SUS that we are building: knowledge and performance of different social actors. Research, Society and Development, 9(3), e122932664. https://doi.org/10.33448/rsd-v9i3.2664. 